\title{
Moth pheromone systems: behavior, biochemistry, peripheral and central neurophysiology
}

\author{
Linda M.Kennedy and Charles E.Linn, $\mathrm{Jr}^{1}$ \\ Department of Biology and Neuroscience Program, Clark University, \\ Worcester, MA 01610 and 'Department of Entomology, Cornell University, \\ New York State Agricultural Experiment Station, Geneva, NY 14456, USA
}

The following five review articles result from a symposium that took place at the Tenth Annual Meeting of the Association for Chemoreception Sciences (AChemS) in Sarasota, Florida, in April 1988, with support from the National Science Foundation (BNS-8719518), E.I.Du Pont de Nemours, Inc., the Provesta Corporation, Clark University and AChemS. The purpose of the symposium was twofold: (i) to stimulate interaction between chemoreception researchers who work with insects and those who work with humans and other vertebrate and invertebrate species, and (ii) to present the moth pheromone system as a model for integrated behavioral/biochemical/ neurophysiological research and for work with mixture stimuli. The moth system is ideal for these goals. During the past five years, significant advances in the behavioral, chemical and physiological aspects have placed this system among the best developed in olfaction. The solutions to several key problems in this system should provide useful information for those involved in similar studies with other vertebrate and invertebrate chemosensory systems. With the same goals, these articles extend the symposium to the Chemical Senses readership. They review the history and development of research on moth pheromone systems, present new data and identify current problems and issues. They should be useful for teaching in courses and for new graduate students as well as for the interdisciplinary exchange of information and ideas.

The symposium presents the story of the moth pheromone work. This Introduction is to tie the aspects of that story together and guide students and other readers who are new to this research. The individual articles concentrate on specific areas from molecular to organismic levels and focus on information relevant to general problems in chemoreception. First, Bjostad (p. 411) discusses a primary problem common in studies of chemosensory systems-that of defining the relevant stimulus. In the case of moth sex pheromones, this problem is that of characterizing the chemical components of the pheromone. The search was limited in the past by the resolution of the equipment. But new chromatography columns so precise as to permit analysis of the pheromone from a single moth, together with the development of a predictive, theoretical approach to biochemical synthesis have led to significant advances. Recent taxonomic studies of the biosynthetic bases for structural features such as double bond position and geometry, chain length and terminal functional group have aided the detection and identification of even trace components in a pheromone blend. It now is possible to determine with considerable accuracy the various components in a particular pheromone blend.

The pheromone produced by a female moth is a mixture of $2-7$ components, which are species-specific in their identity and ratios in the mixture. Male moths respond to 
these pheromone blends to locate, court and copulate with conspecific females. Linn and Roelofs (p. 421) describe the development of understanding about how the pheromone signal controls male behavior and emphasize the importance of the exchange of information between chemical and behavioral investigators. As is the case for natural stimuli in other chemosensory systems, the problem involves analysis of responses to stimulus mixtures. The primary questions concern (i) the sensitivity of a male to the blend, i.e. his ability to detect and discriminate the conspecific blend, and (ii) the functional roles of the pheromone components. It has been shown that a male's behavior involves two points of change-the first for initiation of flight toward the female and the second for abrupt cessation of that flight. These change-points occur at lower and higher pheromone concentrations respectively, and define a spatial area for peak attraction in a matrix of ratio/concentrations of the individual pheromone components. The peak attraction is at component ratios that correspond to the blend released by the conspecific female. There has been confusion, however, about the functions of the pheromone components. Early studies focused on the effectiveness of the major components (one or two for each pheromone blend) in field trapping experiments and attempted to assign the control of individual behaviors in the attraction, courtship, copulation sequence to individual components. But with the development of the more precise chemical and biosynthetic analyses described by Bjostad and of more discriminating laboratory and field behavioral tests, there is new evidence that the entire blend functions as a species-specific unitary signal for long-range attraction, courtship and copulation. Current work focuses on how environmental factors such as temperature (see Baker $e t$ al.) and photoperiod affect male sensitivity to the pheromone. An intriguing question for the future concerns how the pheromone blends function in population dynamics and the evolution of species. Behavioral and neurophysiological data suggest that components may be involved in interspecific behavioral inhibition (see Linn and Roelofs, Grant et al., Christensen $e t$ al.), as well as the species-specific attraction.

An important aspect of chemosensory function involves the orientation mechanisms by which an animal obtains optimal information from, and responds appropriately to a stimulus. For moth pheromone systems, a second focus of behavioral research has been on the relationships between the airborne pheromone plume structure and the way in which males orient and fly upwind along the chemical plume. Recent work has shown that the 'broken, filamentous' structure of the plume and the consequent fluctuating nature of the pheromone stimulation are crucial. Accordingly, Baker et al. (p. 439) used intermittent stimulation to probe mechanisms for the abrupt cessation of male flight toward the female: they recorded receptor cell responses from antennae placed in irregular plumes produced by intermittent puffs of conspecific blends or major components. Responses adapted within 3-5 s to the higher concentrations known to produce cessation of flight, whereas responses to concentrations known to assist mate location did not. Cooling, which produces flight cessation at lower pheromone concentrations, resulted in receptor cell adaptation at lower pulse rates. Interestingly, responses to high concentrations of a second pheromone component did not adapt. Baker et al. suggest that pheromone blend concentrations which produce flight cessation may do so via differential adaptation of receptor cell responses that result in altered afferent information about both overall concentrations and blend ratios. 
After the stimulus has been received, the nervous system must process the olfactory information. The problem here is to understand how stimulus information is encoded and how the nervous system orchestrates the species-specific behaviors. In pursuit of such insight, neurophysiologists have characterized the responses of pheromone-sensitive neurons in male moth peripheral and central nervous systems. They have found that individual antennal neurons respond primarily, but not exclusively, to one or another of the major pheromone components. Yet how receptor cells might encode the species-specific information about minor components in the pheromone blend remains the subject of much discussion. In some instances, responses to minor components have not been found. However, responses to major components are modulated when mixtures of two major or one major and one minor components are presented as stimuli. Future studies of receptor cell responses to selected portions of the ratio/concentration matrices of pheromone components used in the previous behavioral work could be informative. Using a different approach, Grant et al. (p. 449) present new data on the fine structure of responses to repeated stimulus pulses, used to mimic stimulation in a natural pheromone plume. While some cells gave phasic-tonic responses to a single pheromone component, others gave only tonic responses. Interestingly, a portion of the phasic component (first 100-200 ms), when present, was maintained in responses to repeated pulses. In apparent contrast, Baker et al. found adaptation when the numbers of action potentials were counted over 1-s intervals, a measure that would reflect primarily the tonic component. It may be that responses differ for the different species used by the two research groups; indeed differences do occur in the behaviors. Alternatively, it may be that phasic and tonic responses of individual cells, as well as responses of different cells, carry different information. Perhaps future studies focused on moth pheromone-sensitive receptor cell phasic responses to the conspecific blends and components will bring to light some of the elusive mechanisms for component/blend coding.

Moth antennal receptor cells project to the antennal lobes in the brain. These sexually dimorphic lobes are organized into glomeruli, much like vertebrate olfactory bulbs. Male antennal lobes contain a 'macroglomerular complex', where the pheromonesensitive receptor cells terminate. This glomerulus also contains local interneurons and output neurons which project to other brain structures. Christensen et al. (p. 463) describe the responses of macroglomerular cells and relate those responses to the various aspects of the stimulus that are known to control male behavior and also to known receptor cell responses. Their new intracellular data from two species with common living environments and activity cycles and similar but different pheromone blends support and extend earlier data from another species and permit classification of cells into two broad categories. The first, 'blend generalists', respond to all three species' pheromone blends, but not to all of the single components. 'Blend specialists' respond to their conspecific pheromone blends. Some cells also follow pulsed stimuli. Synergism or suppression by mixtures was observed as well. Overall, there appear to be parallel paths of coding, as are known in other insect and vertebrate sensory systems. The paths differ from each other in the types of information for which they code, i.e. the types of information discussed in the preceding articles. Their recognition was dependent upon the conceptual organization that emerged from the previous biochemical, behavioral and receptor cell studies and guided the central nervous system studies. For example, 
the use of pulsed stimuli similar to that used in the Baker et al. and Grant $e t$ al. behavioral and receptor cell studies has revealed 'specialist' cell subtypes that could not have been otherwise shown. Further analyses of the responses of macroglomerular cells and of cells in the brain areas to which they project, together with the continued refinement of knowledge about pheromone chemistry, mate-seeking behaviors, and receptor cell biochemistry and neurophysiology should extend and enhance this remarkable account of chemosensory function.

There are numerous similarities between the moth pheromone systems described in these symposium articles and chemosensory systems in other animals. We hope that the interdisciplinary exchange of information and ideas will stimulate new approaches in both insect and other animal realms of chemosensory research.

Received on July 8, 1988; accepted on February 2, 1989 